Sas, C., O'Grady, M. J., O'Hare, G. M.P., "Electronic Navigation - Some Design Issues", Proceedings of the $5^{\text {th }}$ International Symposium on Human Computer Interaction with Mobile Devices and Services (MobileHCl'03), Udine, Italy, 2003. pp. 471-475. LNCS 2795, Springer Verlag.

\title{
Electronic Navigation - Some Design Issues
}

\author{
Corina Sas, Michael O'Grady \& Gregory O'Hare \\ Practice \& Research in Intelligent Systems \& Media (PRISM), Department of \\ Computer Science, University College Dublin (UCD), Belfield, Dublin 4 \\ Ireland. \\ \{corina.sas, michael.j.ogrady, gregory.ohare\}@ucd.ie
}

\begin{abstract}
Navigation support will form a critical component of future mobile computing systems. However, the ability of people to navigate in unfamiliar environments can vary substantially. In this paper, it is argued that the current generation of mobile devices and associated software does not adequately support the navigational requirements of a broad segment of users and that alternative strategies and design criteria need to be considered.
\end{abstract}

\section{Introduction}

Mobile computing is likely to be the next major computer-usage paradigm. As such, the area remains the focus of much research as advances in pervasive computing, wearable computing and location-aware computing testifies. One service that is considered fundamental in future mobile computing environments is that of navigation support. Such support could encompass an electronic map with the user's position highlighted in real-time or, perhaps, route planning and direction giving.

Researchers have known for some time that males and females adopt different strategies when navigating in the real world [1]. The reason for this difference, possible routed in anthropology, is still unclear but indications are that males have a somewhat higher spatial ability than females. Though navigation aids are starting to proliferate under various guises, there are no indications that the differing spatial abilities of their target population are being considered and addressed. In short, the female population is currently at a disadvantage when using current navigation tools; a disadvantage that may have serious ramifications when carrying out their everyday work. A critical challenge for researchers in mobile HCI is to identify design principles that can compensate for and ultimately eliminate this disadvantage.

In the following sections, the current state-of-the-art in electronic navigation is briefly outlined. After this, Gulliver's Genie, a mobile electronic tourist guide is introduced. The results of evaluations conducted on it are then analysed and discussed in terms of the gender differences that emerged. 


\section{Advances in Electronic Navigation}

Though there exists quite a number of technologies that can be used to determine position, techniques using satellites are by far the most prevalent The Global Positioning System (GPS) is the most popular system at present and is being used in many diverse areas. Reasons for its success include among others, reliability and worldwide coverage. GLONASS, deployed by the Russian Federation, offers similar features to GPS. It is important to remember that both GPS and GLONASS were designed with military applications in mind and remain under the control of their respective defence ministries. More recently, the EU have launched the Galileo initiative, a system oriented towards the needs of civilians and includes safeguards regarding the status and reliability of the positioning signal. Galileo is scheduled to be fully operational by 2008. In the meantime, significant effort is being expended in the area of Satellite Based Augmentation Systems (SBASs) which seek to augment the original GPS signal with addition data leading to more accurate position readings.

An alternative to satellite techniques includes approaches based on the topologies of cellular telecommunication networks. The impetus for research into such techniques came from the FCC's E-911 directive, which stipulates that network operators must be able to pinpoint an emergency call to within 100 meters on average. Technically, deploying networks that utilise these techniques has proved somewhat difficult. However, the next generation or Third Generation (3G) of cellular networks will actively support a number of such techniques.

\section{A Mobile Electronic Tourist Guide}

A portable electronic guide that tourists could carry around with them while exploring a city is regarded by some as an archetypal mobile computing application. A number of prototypes of such systems have been developed in the laboratory, for example, CRUMPET [2] and GUIDE [3]. Likewise, a number of commercial products have also been developed including Vindigo [4] and Port@ble Guide [5].

\subsection{Gulliver's Genie}

Gulliver's Genie [6] was developed to provide navigation and location-aware services to tourists. An additional benefit that it can offer is a better understanding of how tourists act when exploring an outdoor environment. The system is hosted on a PDA which is connected to a set of back-end servers via a wireless data connection.

GPS is used for determining position and a suite of intelligent agents has been developed for anticipating the tourist's requirements. Adaptivity is a key principal underpinning the Genie so all information presented to the tourist has been adapted to their location, orientation and interest profile. 


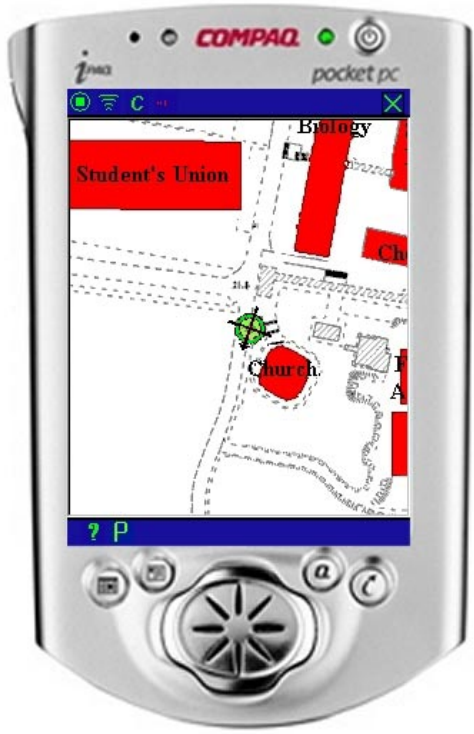

Fig 1: The Genie's navigation screen. In this case the tourist is approaching a church.

Navigation support is delivered via a geocoded electronic map. In essence, this is an electronic image of an appropriately scaled map that has been tagged with such information as facilitates the swift resolution of a GPS position into its corresponding point on the electronic map. The map itself, supplied by the local municipal authority, shows routes and buildings. However, any item that is of likely to be of interest to tourists has been highlighted and labelled (Fig. 1). As the tourist explores their environment, their position and orientation on the map is highlighted and continuously updated. In this way, they can see their position relative to the various attractions at a glance. The option of explicitly scrolling the map is also available.

\subsection{Evaluation}

A thorough user evaluation of the Genie has been carried out here on the campus of University College Dublin. Though relatively modern, the campus contains a number of historical building as well as being adorned with various works of art. Participants were invited to take a tour of the campus with the Genie for about 40 minutes. On completion, a questionnaire was filled in which, amongst other things, tried to ascertain how they rated the Genie as an aid when navigating. The sample group consisted of 40 subjects of which less than half were female (46\%). Though most of the participants had some experience of computing, a small majority (56\%) did not have any prior experience of PDAs. Initial reaction to the Genie was favourable with over $85 \%$ of participants indicating satisfaction with the system. From a navigational perspective, just over half (51\%) identified navigation support as the Genie's most 
important feature. It also emerged that females were significantly more dependant on external aids such as maps $(\mathrm{t}(35)=2.03, \mathrm{p}<0.05)$ and guides $(\mathrm{t}(35)=2.65, \mathrm{p}<0.05)$. To identify differences when using the Genie and to formulate solutions for a better design that would address the specific needs of female users, we investigated both sense of direction and the manner of using the Genie. Without identifying statistically significant differences, the males' sense of direction surpasses the females' sense of direction. In fact, twice as many females (40\%) consider their sense of direction as poor or average, against $21 \%$ of males who gave the same estimation (Fig. 2). With respect to the manner of using the system, we identified a difference regarding the rotation of the device. When asked about the Genie's support for orientation, female users admitted to rotating the PDA so that the map coincided with their own immediate heading significantly more often than males $(t(35)=2.09, \mathrm{p}<0.05)$.

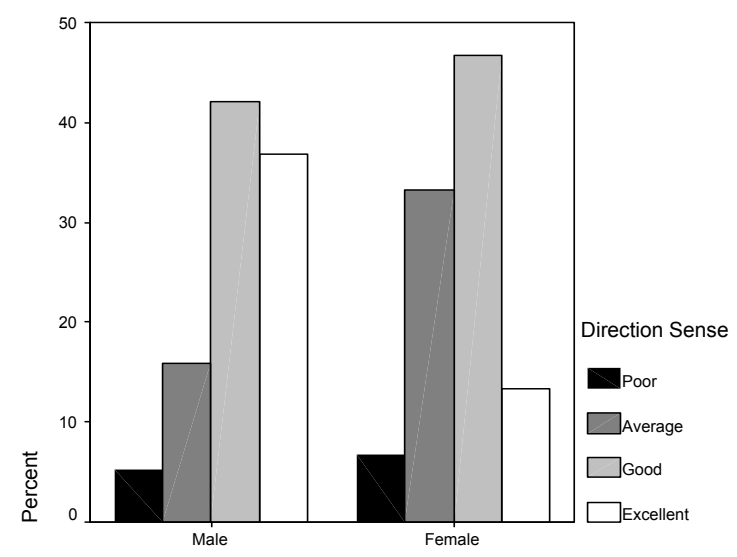

Fig. 2: Users' sense of direction.

\subsection{Implications for Navigation}

Results of the evaluations clearly indicate that users see navigation support as a useful and desirable feature. Findings suggest not only the necessity but also the means for improving system usability to specifically address the needs of female subjects, a significant segment of potential users. The option of being able to ascertain their position at all times was an attractive feature and helped in maintaining good position and orientation awareness. Naturally this provided a comfort and reassurance to some thus facilitating a more proactive and adventurous approach to exploration.

\subsection{Some possible solutions}

Some clues as to how the navigation issue might be addressed have been gleaned from further analysis of the evaluation feedback. 
Automatic map rotation which would match the user's heading: This solution is suggested by the previously presented findings, as well as being mentioned in the users' answers regarding system improvements. This (optional) facility would enable users gain a better spatial awareness and orientation. The position on the map and its corresponding position in the real world would be easily identified thus reducing the cognitive overload associated with the manual rotation of the device.

Improved Position: While GPS enables position readings to within 20 meters on average, this can make the association between one's actual position and that on the electronic map somewhat difficult. If an SBAS can be used, readings of up to five meters in accuracy can be obtained thus improving the situation dramatically. 3D Interface: Augmenting the electronic map with its 3D equivalent was another suggestion as to how the interface might be improved. In this case, the user would have the option of selecting their preferred mode. When using a $3 \mathrm{D}$ interface, the user would have the 3D equivalent of the real world in front of them. However, the success of such an approach is closely related to the availability of more accurate position readings as any divergence between the 3D scene and its geographic equivalent would be even more pronounced than when using a normal 2D map.

\section{Future Work}

An immediate priority is to improve the accuracy of the position readings available to the Genie by using an SBAS, probably the European Geostationary Navigation Overlay Service (EGNOS). Different applications involving 3D representations of real or virtual environments have been developed with the purpose of improving user's navigation. However, few attempts have been concerned with the usability of PDA devices supporting navigational tasks. Tourist attractions could be emphasised by presenting them in a 3D form at the user's request, in the context of a $2 \mathrm{D}$ map. This would enable the users to maintain their orientation due to the bird's eye view afforded by the 2D map, as well as providing additional information on landmarks of interest. Therefore, the development of a mixed 3D landmark representation in the context of 2D maps, is another direction which should be tested by further studies.

\section{References}

1. Halpern, D. F.: Sex Differences in Cognitive Abilities. $3^{\text {rd }}$ edition. Lawrence Erlbaum Associates, Inc. Mahwah, NJ 07430-2262 (2000)

2. Poslad, S., Laamanen, H., Malaka, R., Nick, A., Buckle, P., Zipf, A., "CRUMPET: Creation of User-friendly Mobile Services Personalised For Tourism", In: Second International Conference on 3G Mobile Communication Technologies, London, UK, March 2001.

3. Cheverst, K., Mitchell, K., Davies, N.: The Role of Adaptive Hypermedia in a ContextAware Tourist Guide. Communications of the ACM, Vol. 45 (5) (2002) 47-51.

4. Vindigo, Inc. New York. http://www.vindigo.com

5. Port@ble Internet, Inc. New Jersey, http://www.portableinternet.com/frommers

6. O'Hare, G., O'Grady, M..: Gulliver's Genie: A Multi-Agent System for Ubiquitous and Intelligent Content Delivery. Computer Communications, Vol. 26 (11) (2003), 1177-1187. 